\title{
Deletion of Phytochelatin Synthase Modulates the Metal Accumulation Pattern of Cadmium Exposed C. elegans
}

\author{
Yona J. Essig 1,2, Samuel M. Webb ${ }^{3}$ and Stephen R. Stürzenbaum 1,2,* \\ 1 Analytical and Environmental Sciences Division, Faculty of Life Sciences \& Medicine, \\ King's College London, London SE1 9NH, UK; julie.essig@kcl.ac.uk \\ 2 Medical Research Council-Public Health England (MRC-PHE) Centre for Environment \& Health, \\ King's College London, London SE1 9NH, UK \\ 3 Stanford Synchrotron Radiation Lightsource, SLAC National Accelerator Laboratory, 2575 Sand Hill Road, \\ Menlo Park, CA 94025, USA; samwebb@slac.stanford.edu \\ * Correspondence: stephen.sturzenbaum@kcl.ac.uk; Tel.: +44-207-848-4406
}

Academic Editor: Reinhard Dallinger

Received: 14 January 2016; Accepted: 14 February 2016; Published: 19 February 2016

\begin{abstract}
Environmental metal pollution is a growing health risk to flora and fauna. It is therefore important to fully elucidate metal detoxification pathways. Phytochelatin synthase (PCS), an enzyme involved in the biosynthesis of phytochelatins (PCs), plays an important role in cadmium detoxification. The PCS and PCs are however not restricted to plants, but are also present in some lower metazoans. The model nematode Caenorhabditis elegans, for example, contains a fully functional phytochelatin synthase and phytochelatin pathway. By means of a transgenic nematode strain expressing a pcs-1 promoter-tagged GFP ( $p c s-1:: \mathrm{GFP})$ and a $p c s-1$ specific qPCR assay, further evidence is presented that the expression of the $C$. elegans phytochelatin synthase gene ( $p c s-1)$ is transcriptionally non-responsive to a chronic $(48 \mathrm{~h})$ insult of high levels of zinc $(500 \mu \mathrm{M})$ or acute $(3 \mathrm{~h})$ exposures to high levels of cadmium $(300 \mu \mathrm{M})$. However, the accumulation of cadmium, but not zinc, is dependent on the pcs-1 status of the nematode. Synchrotron based X-ray fluorescence imaging uncovered that the cadmium body burden increased significantly in the pcs-1(tm1748) knockout allele. Taken together, this suggests that whilst the transcription of pcs-1 may not be mediated by an exposure zinc or cadmium, it is nevertheless an integral part of the cadmium detoxification pathway in C. elegans.
\end{abstract}

Keywords: C. elegans; nematode; phytochelatin synthase; X-ray fluorescence microscopy

\section{Introduction}

Phytochelatin (PC), a non-ribosomal heavy-metal complexing peptide, was first identified in monocot and dicot plants [1]. They are characterized by a repetitive ( $\gamma$-GluCys) ${ }_{n}$ Gly motif (where $n$ typically ranges from 2 to 5 ) and their ability to bind to cadmium and other non-essential metals (e.g., arsenic and mercury) but also essential heavy metals (such as copper and zinc) via cysteine thiol residues [1]. PCs are biosynthesised by the enzyme glutathione gamma-glutamylcysteinyltransferase, which was isolated in 1989 from Silene cucubalus, a plant species belonging to the Caryophyllaceae family [2], and thus was named phytochelatin synthase (PCS). The phytochelatin nomenclature is however misleading, as PCs and PCS have since been identified in numerous taxonomic groups of the (lower) animal kingdom, including free-living nematodes, parasitic nematodes, flatworms, segmented worms and molluscs (Table 1) and therefore PCS is also frequently referred to as a glutathione gamma-glutamylcysteinyltransferase, to reflect its function rather than phylogenetic origin. Based on 
sequence homology, the $\mathrm{N}$-terminal is more conserved than the C-terminal. It has been suggest that the $\mathrm{N}$-terminal is may be linked to the activity of the enzyme and the $\mathrm{C}$-terminal might be important for the regulation of the enzyme but not for the activity [3].

Table 1. Phytochelatin synthases/glutathione gamma-glutamylcysteinyltransferases identified within the Kingdom Animalia.

\begin{tabular}{|c|c|c|c|}
\hline \multicolumn{4}{|c|}{ Phylum Nematoda (Nematodes) } \\
\hline Class & Order & Species & Accession number \\
\hline Chromadorea & Rhabditida & Caenorhabditis elegans & AF299333.1 \\
\hline Chromadorea & Rhabditida & Pristionchus pacificus & ABKE02006821 \\
\hline Chromadorea & Spirurida & Loa loa & XM_003138991.1 \\
\hline Chromadorea & Ascaridida & Ascaris suum & AEUI02000059.1 \\
\hline Secernentea & Rhabditida & Caenorhabditis briggsae & FR847113.2 \\
\hline Secernentea & Rhabditida & Caenorhabditis remanei & DS268408.1 \\
\hline Secernentea & Rhabditida & Caenorhabditis brenneri & XM_003117257.1 \\
\hline Secernentea & Rhabditida & Haemonchus contortus & HF966434.1 \\
\hline Secernentea & Strongylida & Ancylostoma ceylanicum & KE125293.1 \\
\hline Secernentea & Strongylida & Necator americanus & XM_013449818.1 \\
\hline Secernentea & Strongylida & Ancylostoma duodenale & KN7734493.1 \\
\hline Secernentea & Strongylida & Oesophagostomum dentatum & KN567239.1 \\
\hline Secernentea & Strongylida & Ancylostoma ceylanicum & KC914882 \\
\hline Secernentea & Spirurida & Brugia malayi & XM_001902065.1 \\
\hline Secernentea & Ascaridida & Toxocara canis & JPKZ01001678.1 \\
\hline \multicolumn{4}{|c|}{ Phylum Annelida (Segmented Worms) } \\
\hline Class & Order & Species & Accession number \\
\hline Clitellata & Haplotaxida & Lumbricus rubellus & $\begin{array}{c}\text { KC981075.1 / } \\
\text { КС981074.1 }\end{array}$ \\
\hline Clitellata & Haplotaxida & Eisenia fetida & EF433776.1 \\
\hline Clitellata & Haplotaxida & Eisenia andrei & KP770990.1 \\
\hline Clitellata & Rhynchobdellida & Helobdella robusta & XM_009013566 \\
\hline Polychaeta & Capitellida & Capitella teleta & KB309928 \\
\hline \multicolumn{4}{|c|}{ Phylum Gastropoda (Molluscs) } \\
\hline Class & Order & Species & Accession number \\
\hline Gastropoda & Lottiida & Lottia gigantea & XM_009047767 \\
\hline Bivalvia & Ostreoida & Crassostrea gigas & XM_011447075 \\
\hline \multicolumn{4}{|c|}{ Phylum Platyhelminthes (flatworms) } \\
\hline Class & Order & Species & Accession number \\
\hline Trematoda & Strigeidida & Schistosoma mansoni & CABG01000042.1 \\
\hline Trematoda & Prosostomata & Schistosoma haematobium & XM_012938931.1 \\
\hline Trematoda & Opisthorchiida & Opisthorchis viverrini & XM_009168655 \\
\hline Trematoda & Opisthorchiida & Clonorchis sinensis & DF143054 \\
\hline Cestoda & Cyclophyllidea & Echinococcus multilocularis & LK031422.1 \\
\hline Cestoda & Cyclophyllidea & Echinococcus granulosus & LK028580.1 \\
\hline Cestoda & Cyclophyllidea & Hymenolepis microstoma & LK053266.1 \\
\hline
\end{tabular}

Clemens et al. [4] and Vatamaniuk et al. [5] discovered the presence of a functional phytochelatin synthase in the model nematode Caenorhabditis elegans, now one of the most studied metazoan phytochelatin synthases. The fully sequenced $C$. elegans genome contains two pcs-1 splice variants encoding a 426aa and 418aa protein, respectively, which are approximately $30 \%$ identical to the Arabidopsis thaliana PCS [3]. Knocking out or knocking down (by RNA interference) the C. elegans pcs-1 gene results in a hypersensitive $\mathrm{Cd}^{2+}$ phenotype [5,6], leading to developmental retardation and early death. Intriguingly, the exposure to cadmium increases the concentration of PCs in the tissue of C. elegans, but pcs-1 is notably transcriptionally non-responsive to metal exposures [6], a finding which 
was also observed in earthworms [7]. Although the PCS/PC pathway has been linked to cadmium detoxification $[7,8]$, it has, to date, not been possible to provide evidence that the impairment of the phytochelatin synthase pathway influences directly the accumulation of metals in animals, a notion this study aimed to investigate.

The transcription of $C$. elegans $p c s-1$ was quantified in animals challenged with high levels of cadmium or zinc by means of in vivo imaging using transgenic nematodes expressing a Ppcs-1::GFP and by quantitative RT-PCR. In addition, synchrotron based X-ray fluorescence imaging was optimized to facilitate the measurement of the cadmium and zinc body burden in individual nematodes. The latter enabled the means to assess to what extent metal load changes in wild-type and pcs-1 knockout nematodes raised in the presence or absence of high levels of cadmium or zinc, thereby providing first tantalizing insights into the pcs-1 mediated alteration in metal accumulation.

\section{Results and Discussion}

Cadmium and zinc are chemically similar and are both positioned in group IIb of the periodic table. Two electrons are found on their most outer shell, hence the valencies of both elements is " $2+$ ". However, the affinity towards -SH groups is higher in the case of cadmium, which is an important fact given that cadmium is a toxic, non-essential metal, but zinc essential to a myriad of proteins and enzymes. Indeed, metal ions often interact and it is therefore important to define their precise biological function within biological systems, and in particular individuals with genetic variations within key metallo-pathways [9]. Defining the interaction between cadmium and zinc within an organism is therefore deemed to be crucial and has been nicely reviewed by Brzoska and Moniuszko-Jakoniuk [10].

The difference in chronic and acute exposure conditions were due to the different toxicities of the respective metals (based on the molar concentration zinc is less toxic to nematodes than cadmium, indeed exposure to $300 \mu \mathrm{M}$ cadmium for extended time periods kills the nematode) and the need to apply the same exposure conditions to all experimental platforms (a relatively high concentration of metal is required to obtain a sufficiently strong signal in individual nematodes to allow the detection by X-ray fluorescence microscopy, see later). The concentrations of cadmium is high, but within the same order of magnitude as reported in Cui et al. [11], who exposed C. elegans to $100 \mu \mathrm{M}$ for up to $36 \mathrm{~h}$ or $200 \mu \mathrm{M}$ cadmium for $24 \mathrm{~h}$. Cui et al. [11] were not able to observe a significantly change the expression of pcs-1 at these concentrations and to confirm that this was not merely due to a threshold effect, we decided to determine whether an exposure to higher levels of cadmium (300 $\mu \mathrm{M})$ or zinc $(500 \mu \mathrm{M})$ may influence the expression of phytochelatin synthase. It should however be noted that although transcription did not change at the stated concentrations of zinc or cadmium, it is conceivable that an exposure to lower concentrations may induce the transcription of $p c s-1$.

\subsection{Analysis of Ppcs-1::GFP Expression by Fluorescence Microscopy}

The expression of pcs-1 was quantified, in vivo, utilizing a transgenic C. elegans which bears extra-chromosomal copies of the pcs-1 promoter fused to the coding sequence of the Green Fluorescent Protein, GFP (Ppcs-1::GFP). The constitutive expression of Ppcs-1::GFP was confirmed, as reported in $[12,13]$ and shown to be limited to the cells of the pharyngeal intestinal valve and the rectal valve (Figure 1A) and whilst we are not able to hypothesize why the GFP signal was higher in the pharyngeal intestinal valve than in the rectal calve, quantitative assessment confirmed that the fluorescence intensity did not change within the two locations in nematodes exposed to $500 \mu \mathrm{M}$ zinc or $300 \mu \mathrm{M}$ cadmium (Figure 1B). The analysis of GFP signal intensity in individual nematodes suggests that the transcription of $p c s-1$ is not dependent on the exposure to cadmium or zinc, at least at the concentrations indicated. 
A

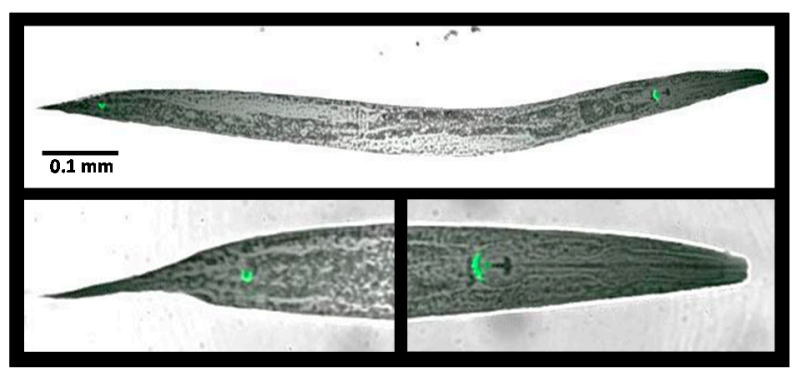

$\mathbf{B}$
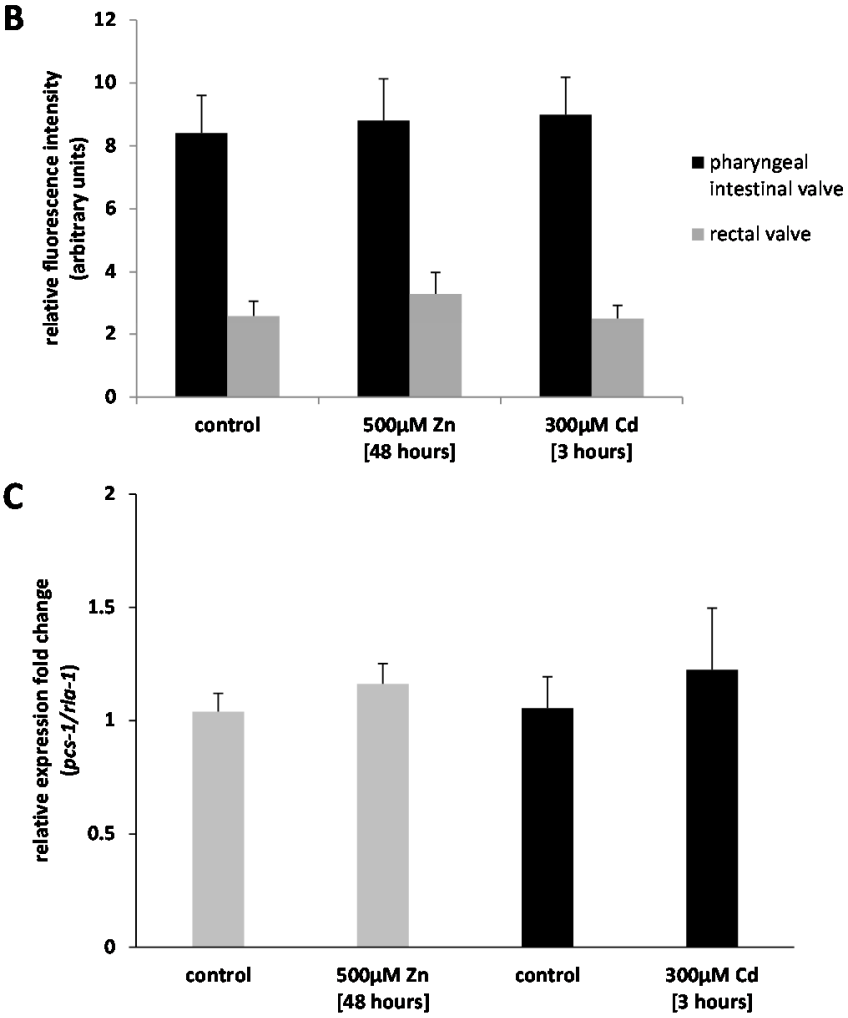

Figure 1. The transcriptional activity of the $p c s-1$ promoter. The expression pattern of the $p c s-1$ promoter was visualized in Ppcs-1::GFP transgenic nematodes (synchronized at L4 stage) by means of confocal microscopy (Leica DMIRE2) (A); fluorescence in the head and tail region was quantified using ImageJ (B); Note, the fluorescence intensity, though universally higher in the pharyngeal intestinal valve compared to the intensity in the rectal valve, were statistically indistinguishable in nematodes raised under control conditions or nematodes exposed to $500 \mu \mathrm{M}$ zinc for $48 \mathrm{~h}$ or $300 \mu \mathrm{M}$ cadmium for $3 \mathrm{~h}$. The error bars represent the standard error of the mean $( \pm$ SEM, biological repeats $n=9)$. A quantitative RT-PCR confirmed the transcriptional invariance of $p c s-1$ in wild-type nematodes challenged with metals (C). The error bars denote \pm SEM (technical repeats $n=3$, biological repeats $n=3$ ). Statistical analyses were performed using a one way ANOVA). Note, P pcs-1::GFP fluorescence signal intensity or $p c s-1$ transcription did not differ $(p>0.05)$ in control and metal exposed nematodes.

\subsection{Analysis of PCS-1 Expression by Quantitative PCR}

To independently validate the results obtained in the GFP assay, the expression fold-change of pcs-1 was determined by qPCR in wild-type (N2) nematodes exposed to either $500 \mu \mathrm{M}$ zinc for $48 \mathrm{~h}$ or $300 \mu \mathrm{M}$ cadmium for $3 \mathrm{~h}$, respectively. Exposures were conducted as true biological replicates, and synchronized (fourth larval stage, L4) nematodes harvested that had either not been subjected to metals, acutely dosed with cadmium or chronically exposed to zinc. Following normalization with the invariant ribosomal rla-1, analysis confirmed that the transcriptional response to metal exposure was, at best, negligible. In detail, nematodes challenged with $500 \mu \mathrm{M}$ zinc for $48 \mathrm{~h}$ prior to sample 
collection resulted in a 1.2-fold increase in pcs-1 and $300 \mu \mathrm{M}$ cadmium for $3 \mathrm{~h}$ in a 1.8-fold increase, both values are statistically not different to the baseline levels observed in the control exposures (Figure 1C). Metallothionein ( $m t l-2)$ was utilized as a positive control to validate that the cDNA synthesized originated from successful exposures. The $m t l-2$ transcript increased 5.2-fold and $m t l-1$ transcript 3.5-fold in the respective cadmium and zinc exposed samples, which confirmed that the lack of $p c s-1$ response was genuine and not due to a failed exposure. These results not only confirm the results obtained using the Ppcs-1::GFP strain, but substantiate that even high (near toxic) levels of zinc or cadmium are not able to activate the transcription of $p c s-1$ in nematode and extends previous findings by [14-16].

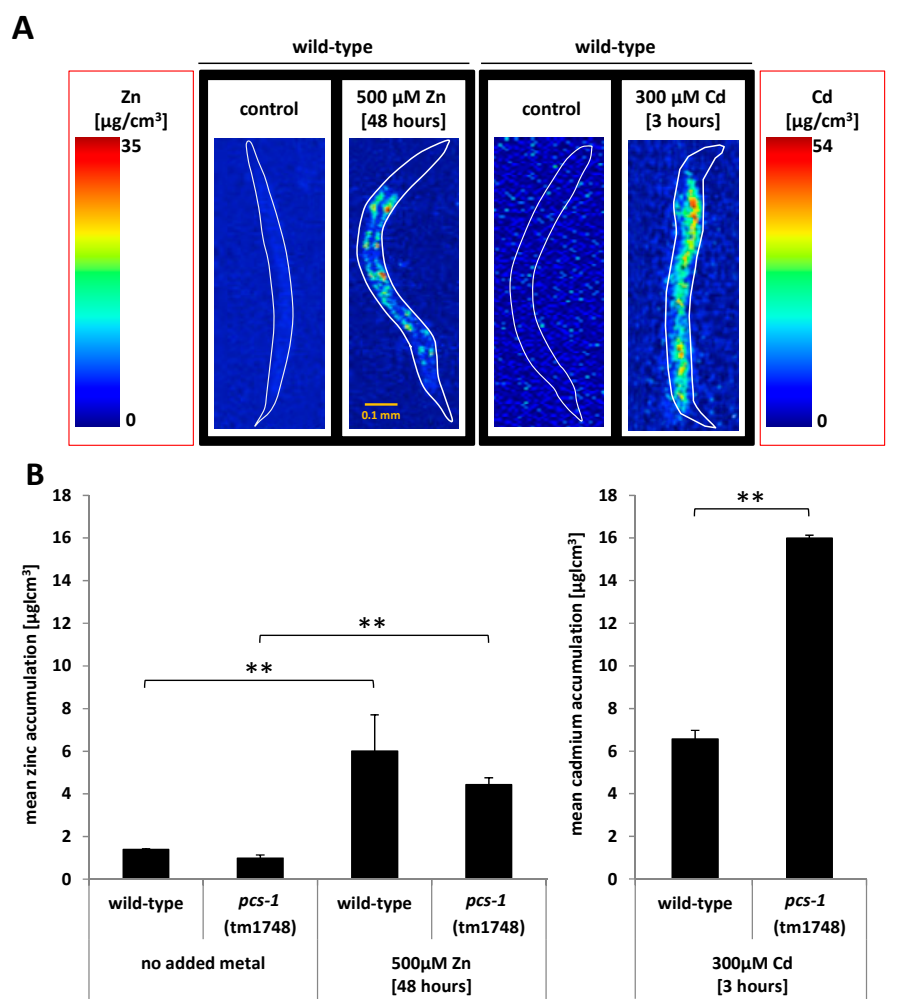

Figure 2. Accumulation of metals in the nematode body. X-ray fluorescence imaging (XFI) was utilized to assess the distribution and accumulation of zinc and cadmium in the body of C. elegans raised either on control plates or transferred to plates supplemented with $500 \mu \mathrm{M}$ zinc for $48 \mathrm{~h}$ or $300 \mu \mathrm{M}$ cadmium for $3 \mathrm{~h}$ (A); Note, base-line levels of zinc (but not cadmium) were observed in nematodes raised on control plates. All quantitative analyses of metal load within the body of nematodes were performed using the MicroAnalysis Toolkit $(\mathbf{A}, \mathbf{B})$. A highly significant difference $(p=0.002)$ was apparent in nematodes exposed to zinc ( $500 \mu \mathrm{M}$ zinc for $48 \mathrm{~h}$ ), however no significant difference was observed between the strains (wild-type and the $p c s-1(\operatorname{tm} 1748)$ mutant). Likewise, exposure to cadmium resulted in a highly significant $(p=0.002)$ increase in metal load in wild-type and $p c s-1(\operatorname{tm} 1748)$. However, strain specific differences were also observed, where the $p c s-1(\operatorname{tm} 1748)$ accumulated significantly $(p=0.002)$ more cadmium than the respective wild-type (B). Note: whilst zinc could be measured in nematodes raised on control plates, the cadmium signal was below the detection limit. Statistical analyses were performed using a factorial ANOVA. Note, the pixel densities differ because $\mathrm{Cd}$ and $\mathrm{Zn}$ quantifications were performed at different beamlines (Cd: 14-3; Zn 2-3), different incident energies (Cd: $3.575 \mathrm{keV}$; Zn $10 \mathrm{keV})$, and different spot sizes (Cd: $5 \times 5 \mu \mathrm{M} ; \mathrm{Zn} 2 \times 2 \mu \mathrm{M})$. ${ }^{* *}$ denotes $p \leqslant 0.01$.

\subsection{Analysis of Metal Accumulation by X-ray Fluorescence Imaging}

To determine the accumulation and distribution pattern of zinc and cadmium within single C. elegans, high resolution X-ray fluorescence imaging (XFI) was applied using the facilities at the 
Stanford Synchrotron Radiation Lightsource (SSRL). Initial trials confirmed that a strong base-line and exposure signals could be obtained from nematodes chronically exposed to zinc, however the chronic exposure to lower concentrations of cadmium $(30 \mu \mathrm{M})$ did not return a signal above the detection limit. Therefore a higher concentration of cadmium, albeit via a more acute exposure route, was chosen. The detection limit requirements of XFI thus dictated the exposure conditions of the experiment conducted in this paper, namely $300 \mu \mathrm{M}$ cadmium for $3 \mathrm{~h}$ and $500 \mu \mathrm{M}$ zinc for $48 \mathrm{~h}$. Wild-type (N2) and the pcs-1 knock out mutant (pcs-1(tm1748)) were exposed to cadmium and zinc using the identical exposure conditions as in the previous experiments and the metal concentration was visualised in individual nematodes by means of a heat map scaled from 0 (blue) to $54 \mu \mathrm{g} / \mathrm{cm}^{3}$ (red) for cadmium and 0 (blue) to $35 \mathrm{\mu g} / \mathrm{cm}^{3}$ (red) for zinc (Figure 2A). Although no additional zinc was added to the control plates or the bacteria, nematodes were seemingly able to obtain sufficient levels of zinc from the media to maintain essential baseline levels of zinc, as evidenced by the detection of a zinc signal (compared to the absence of cadmium signal) in control conditions. Following the exposure to $500 \mu \mathrm{M}$ zinc, a significant accumulation was observed in the intestinal cells of nematodes (Figure 2A). It should be noted that this signal is not free zinc located in the lumen of the gut (as this was cleared prior to analysis) but a genuine signal originating from an uptake of zinc. The presence or absence of the gene pcs-1 did not affect the accumulation pattern or concentration of zinc in nematodes. This suggests that the phytochelatin synthase is not involved in zinc sensing or detoxification, at least at a concentration of $500 \mu \mathrm{M}$ zinc, and contrasts studies in plants which have shown that zinc exposure can influence the phytochelatin synthase [1,17]. During the course of acute dosing, cadmium also increased throughout the nematodes body. However, unlike zinc, a considerable difference in cadmium accumulation was observed between wild-type and the $p c s-1(\mathrm{tm} 1748)$ knock out strain, namely a significant cadmium bio-concentration (wild-type: $\left.(6.6 \pm 0.4) \mu \mathrm{g} / \mathrm{cm}^{3} ; p c s-1(\operatorname{tm} 1748):(16.0 \pm 0.1) \mu \mathrm{g} / \mathrm{cm}^{3}\right)$ was observed upon deletion of the phytochelatin (Figure $2 \mathrm{~B}$ ).

\section{Materials and Methods}

\subsection{Maintenance and Strains}

C. elegans were maintained at $20^{\circ} \mathrm{C}$ on Nematode Growth Media (NGM) $[9,18]$ plates seeded with Escherichia coli OP50 [19]. Three different strains were used, namely the wild-type N2 Bristol strain and the knock out strain pcs-1(tm1748) originally generated by the Mitani laboratory, Tokyo Women's Medical University, Japan (both strains were obtained from the Caenorhabditis Genetics Center stock collection at the University of Minnesota, St. Paul, MN, USA), and UL1109 a transgenic strain carrying a Ppcs-1::GFP extrachromosomal construct (kindly donated by Prof. I. Hope, Leeds University, Leeds, UK). The $p c s-1(\mathrm{tm} 1748)$ is deemed to be a genuine null-allele [13].

\subsection{Metal Exposures}

Metals (500 $\mu \mathrm{M}$ zinc or $300 \mu \mathrm{M}$ cadmium) were added to the molten NGM agar as well as the OP50. The OP50 was spread onto NGM plates which were then incubated at room temperature overnight to allow the formation of a bacterial lawn. The chronic zinc exposure samples were generated by placing synchronized L1 nematodes onto NGM plates containing either no added zinc or $500 \mu \mathrm{M}$ zinc, and then incubated at $20^{\circ} \mathrm{C}$ for $48 \mathrm{~h}$ (until the nematodes had developed into pre-adult L4 stage). The acute cadmium exposures required staged L1 nematodes to be plated initially onto non-metal supplemented plates (control plates) for $45 \mathrm{~h}$ and then transferred to plates containing $300 \mu \mathrm{M}$ for $3 \mathrm{~h}$.

\subsection{Confocal Fluorescence Microscopy}

To visualize and quantify the expression of the pcs-1 transcript, strain UL1109 was used which carries an extrachromosomal Ppcs-1::GFP construct (1543 bp of the pcs-1 promoter and $851 \mathrm{bp}$ of the pcs-1 coding sequence (spanning the first three exons and introns) fused to the coding sequence of GFP) made by Gateway recombination using pDEST-R4R2 and a GFP entry clone with pRF4 (rol-6) serving 
as co-transformant. The transgenic nematodes were maintained on control plates (with no added metals) or exposed to either $500 \mu \mathrm{M}$ zinc for $48 \mathrm{~h}$ or $300 \mu \mathrm{M}$ cadmium for $3 \mathrm{~h}$. L4 nematodes were picked into a drop of M9 and sodium azide (to immobilize the nematode) on a glass slide and imaged using a confocal Leica DMIRE2 microscope (Leica Microsystems, Milton Keynes, UK); fluorescence was captured by using an argon laser $\left(\lambda_{\mathrm{ex}}: 488\right)$ and the quantitative analysis was performed by means of Image $(n=9)$.

\subsection{Quantitative PCR ( $q P C R)$}

To determine the expression of $p c s-1$ by quantitative PCR, each condition/replicate consisted of 7000 synchronized L4 stage C. elegans, which were washed off NGM plates and collected in $15 \mathrm{~mL}$ centrifuge tubes. To eliminate the Escherichia coli OP50, nematodes were washed with M9 buffer four times, and then pelleted, shock frozen in liquid nitrogen and stored at $-80^{\circ} \mathrm{C}$ for at least $24 \mathrm{~h}$. RNA was extracted using the standard Tri Reagent ${ }^{\circledR}$ (Sigma-Aldrich, St. Louis, MO, USA) protocol, bar one modification, namely the frozen nematode pellet with Tri-reagent was vortexed with an equal quantity of acid-washed glass beads (particle size 425-600 $\mu \mathrm{M}$, Sigma-Aldrich) for $4 \mathrm{~min}$. The concentration and integrity of total RNA was determined with a NanoDrop 1000 Spectrophotometer (NanoDrop Technologies, Inc., Wilmington, DE, USA) and by agarose gel (2\%) electrophoresis.

cDNA was synthesised from 1000 ng RNA using an oligo dT primer (5'-(T)20VN-3') and M-MLV reverse transcriptase (Promega, Southampton, UK) applying standard incubation condition. The quantity of $p c s-1$ was measured on an ABI Prism 7500 Fast (Applied Biosystems ${ }^{\circledR}$, Paisley, UK) utilizing the following cycling conditions: $2 \mathrm{~min}$ at $50{ }^{\circ} \mathrm{C}, 10 \mathrm{~min}$ at $95^{\circ} \mathrm{C}$, followed by 40 cycles of $15 \mathrm{~s} 95^{\circ} \mathrm{C}$ and $1 \mathrm{~min} 60^{\circ} \mathrm{C}$. The housekeeping gene rla-1 (acidic ribosomal subunit protein $\mathrm{P} 1$ ) was used for normalisation purposes, as it was previously shown to be invariant in nematodes subjected to a metal exposure $[18,20]$. All probes and primers were designed to be compatible with the Universal Probe Library (Roche Applied Sciences, Burgess Hill, UK). Each qPCR reaction contained $5 \mu \mathrm{L}$ ROX Buffer (Roche Applied Sciences), $0.1 \mu \mathrm{L}$ Probe ( $p c s-1$ probe \#159 or rla-1 probe \#162), $0.4 \mu \mathrm{L}$ forward and reverse primers (pcs-1: forward $5^{\prime}$-AAGCGCCGTGGAGATTCTA- $3^{\prime}$ and reverse $5^{\prime}$-TATTTTCCAAAGGCACACAACA- $3^{\prime}$ or $r l a-1$ : forward $5^{\prime}$-ACGTCGAGTTCGAGCCATA- $3^{\prime}$ and reverse $5^{\prime}$-GAAGTGATGAGGTTCTTCAC-3') and $2 \mu \mathrm{L}$ diluted cDNA $(100 \mathrm{ng} / \mu \mathrm{L})$. The total volume was made up to $10.8 \mu \mathrm{L}$ with nuclease free water. The $\mathrm{CT}$ values were determined using the 7500 Fast System SDS Software (Applied Biosystems ${ }^{\circledR}$ ) and the fold changes in gene expression were calculated by applying the $2^{-\Delta \Delta C t}$ method. Statistical analysis was performed on three independent biological replicates, each consisting of three technical repeat measurements.

\subsection{X-ray Fluorescence Imaging (XFI)}

The zinc and cadmium accumulated in the body of the nematode was visualized and quantified by X-ray fluorescence imaging (XFI) at the Stanford Synchrotron Radiation Laboratory (SSRL). C. elegans wild-type and pcs-1 knockout (pcs-1(tm1748)) strains were placed, at room temperature, into a drop of sodium azide on a metal-free plastic microscope slide. For zinc measurements, beam line 2-3 was used, with an incident energy of $10 \mathrm{keV}$, selected with a $\mathrm{Si}(111)$ double crystal monochromator. The X-ray beam was focused to a $2 \times 2 \mu \mathrm{m}$ spot size using a Rh coated Kirkpatrick-Baez (KB) mirror pair (Xradia Inc (now Zeiss), Pleasanton, CA, USA). The nematodes were rastered in a continuous motion across the beam and the intensity of the fluorescence lines of the elements of interest from the sample were monitored at each pixel using a silicon drift Vortex detector (Hitachi, Northridge, CA, USA) equipped with Xspress3 electronics (Quantum Detectors). Dwell time on an individual pixel was approximately on the order of $75 \mathrm{~ms}$. Cadmium measurements were performed at beam line 14-3, with the major differences being that the incident energy was selected to be $3.575 \mathrm{keV}$, the KB focussing mirrors were $\mathrm{Ni}$ coated, and the $\mathrm{X}$-ray spot size was $5 \times 5 \mu \mathrm{m}$. The resulting images were analysed with the Micro Analysis Toolkit (Webb 2011, Palo Alto, CA, USA). Elemental concentrations were determined by calibration with standard X-ray fluorescence concentration thin film standards 
(MicroMatter, Vancouver, BC, Canada) and lines added to indicate the shape of the nematode. The latter was generated by taking the phosphorous and potassium signals as the template which is ubiquitous throughout the body of the nematode.

\subsection{Statistical Analysis}

Statistical analysis was performed using IBM SPSS. The qPCR was assessed by a one way ANOVA, the fluorescence microscopy was analysed using an independent $t$-test and the XFM was scrutinized via a factorial ANOVA. All error bars represent standard errors of the mean (SEM).

\section{Conclusions}

In conclusion, this paper substantiates the notion that pcs-1 transcription is not activated by zinc or cadmium, even at high concentrations. The finding that the cadmium body burden increases in nematodes characterized by a defective $p c s-1 /$ PC pathway, suggests that the phytochelatin synthase and possibly phytochelatin may actively be involved in the binding, transport, excretion and/or detoxification of cadmium, rather than zinc.

Acknowledgments: We acknowledge funding through King's College London (to SRS) and thank the King's Genomics Centre for access to equipment. Use of the Stanford Synchrotron Radiation Lightsource, SLAC National Accelerator Laboratory, is supported by the U.S. Department of Energy, Office of Science, Office of Basic Energy Sciences under Contract No. DE-AC02-76SF00515. The SSRL Structural Molecular Biology Program is supported by the DOE Office of Biological and Environmental Research, and by the National Institutes of Health, National Institute of General Medical Sciences (including P41GM103393). The contents of this publication are solely the responsibility of the authors and do not necessarily represent the official views of NIGMS or NIH. Finally, we acknowledge Gladio Giannitelli and Garyfalia Vouloutsi, two MSc students who contributed towards the initial optimization of XFM technique with nematodes.

Author Contributions: Stephen R. Stürzenbaum conceived the study and Yona J. Essig performed the experiments. Yona J. Essig, Samuel M. Webb and Stephen R. Stürzenbaum designed the experiments, analyzed the data and wrote the paper.

Conflicts of Interest: The authors declare no conflict of interest.

\section{References}

1. Grill, E.; Winnacker, E.L.; Zenk, M.H. Phytochelatins: The principal heavy-metal complexing peptides of higher plants. Science 1985, 230, 674-676. [CrossRef] [PubMed]

2. Grill, E.; Loffler, S.; Winnacker, E.L.; Zenk, M.H. Phytochelatins, the heavy-metal-binding peptides of plants, are synthesized from glutathione by a specific $\gamma$-glutamylcysteine dipeptidyl transpeptidase (phytochelatin synthase). Proc. Natl. Acad. Sci. USA 1989, 86, 6838-6842. [CrossRef] [PubMed]

3. Rea, P.A.; Vatamaniuk, O.K.; Rigden, D.J. Weeds, worms, and more. Papain's long-lost cousin, phytochelatin synthase. Plant Physiol. 2004, 136, 2463-2474. [CrossRef] [PubMed]

4. Clemens, S.; Schroeder, J.I.; Degenkolb, T. Caenorhabditis elegans expresses a functional phytochelatin synthase. Eur. J. Biochem. 2001, 268, 3640-3643. [CrossRef] [PubMed]

5. Vatamaniuk, O.K.; Bucher, E.A.; Ward, J.T.; Rea, P.A. A new pathway for heavy metal detoxification in animals. Phytochelatin synthase is required for cadmium tolerance in Caenorhabditis elegans. J. Biol. Chem. 2001, 276, 20817-20820. [CrossRef] [PubMed]

6. Hughes, S.L.; Bundy, J.G.; Want, E.J.; Kille, P.; Sturzenbaum, S.R. The metabolomic responses of Caenorhabditis elegans to cadmium are largely independent of metallothionein status, but dominated by changes in cystathionine and phytochelatins. J. Proteom. Res. 2009, 8, 3512-3519. [CrossRef] [PubMed]

7. Bundy, J.G.; Kille, P. Metabolites and metals in Metazoa-What role do phytochelatins play in animals? Metallomics 2014, 9, 1576-1582. [CrossRef] [PubMed]

8. Howden, R.; Andersen, C.R.; Goldsbrough, P.B.; Cobbett, C.S. A cadmium-sensitive, glutathione-deficient mutant of Arabidopsis thaliana. Plant Physiol. 1995, 107, 1067-1073. [CrossRef] [PubMed]

9. Maret, W. The metals in the biological periodic system of the elements: Concepts and conjectures. Int. J. Mol. Sci. 2016. [CrossRef] [PubMed] 
10. Brzoska, M.M.; Moniuszko-Jakoniuk, J. Interactions between cadmium and zinc in the organism. Food Chem. Toxicol. 2001, 39, 967-980. [CrossRef]

11. Cui, Y.; McBride, S.; Boyd, W.; Alper, S.; Freedman, J. Toxicogenomic analysis of Caenorhabditis elegans reveals novel genes and pathways involved in the resistance to cadmium toxicity. Genome Biol. 2007, 8, 90-105. [CrossRef] [PubMed]

12. Hope, I.A.; Stevens, J.; Garner, A.; Hayes, J.; Cheo, D.L.; Brasch, M.A.; Vidal, M. Feasibility of genome-scale construction of promoterreporter gene fusions for expression in Caenorhabditis elegans using a multisite gateway recombination system. Genome Res. 2004, 14, 2070-2075. [CrossRef] [PubMed]

13. Schwartz, M.S.; Benci, J.L.; Selote, D.S.; Sharma, A.K.; Chen, A.G.; Dang, H.; Fares, H.; Vatamaniuk, O.K. Detoxification of multiple heavy metals by a half-molecule ABC transporter, HMT-1, and coelomocytes of Caenorhabditis elegans. PLoS ONE 2010, 5. [CrossRef] [PubMed]

14. Vatamaniuk, O.K.; Mari, S.; Lu, Y.P.; Rea, P.A. AtPCS1, a phytochelatin synthase from Arabidopsis: Isolation and in vitro reconstitution. Proc. Natl. Acad. Sci. USA 1999, 96, 7110-7115. [CrossRef] [PubMed]

15. Clemens, S.; Kim, E.J.; Neumann, D.; Schroeder, J.I. Tolerance to toxic metals by a gene family of phytochelatin synthases from plants and yeast. EMBO J. 1999, 18, 3325-3333. [CrossRef] [PubMed]

16. Ha, S.B.; Smith, A.P.; Howden, R.; Dietrich, W.M.; Bugg, S.; O'Connell, M.J.; Goldsbrough, P.B.; Cobbett, C.S. Phytochelatin synthase genes from Arabidopsis and the yeast Schizosaccharomyces pombe. Plant Cell 1999, 11, 1153-1164. [CrossRef] [PubMed]

17. Grill, E.; Winnacker, E.L.; Zenk, M.H. Phytochelatins, a class of heavy-metal-binding peptides from plants, are functionally analogous to metallothioneins. Proc. Natl. Acad. Sci. USA 1987, 84, 439-443. [CrossRef] [PubMed]

18. Polak, N.; Read, D.S.; Jurkschat, K.; Matzke, M.; Kelly, F.J.; Spurgeon, D.J.; Sturzenbaum, S.R. Metalloproteins and phytochelatin synthase may confer protection against zinc oxide nanoparticle induced toxicity in Caenorhabditis elegans. Comp. Biochem. Physiol. C 2014, 160, 75-85. [CrossRef] [PubMed]

19. Brenner, S. The genetics of Caenorhabditis elegans. Genetics 1974, 77, 71-94. [PubMed]

20. Swain, S.C.; Keusekotten, K.; Baumeister, R.; Stürzenbaum, S.R. C. elegans metallothioneins: New insights into the phenotypic effects of cadmium toxicosis. J. Mol. Biol. 2004, 341, 951-959. [CrossRef] [PubMed]

(C) 2016 by the authors; licensee MDPI, Basel, Switzerland. This article is an open access article distributed under the terms and conditions of the Creative Commons by Attribution (CC-BY) license (http://creativecommons.org/licenses/by/4.0/). 